\title{
EFFECTIVE ELASTIC PROPERTIES OF TEXTURED CUBIC POLYCRYSTALS
}

\author{
E.A. MITYUSHOV*, S.A. BERESTOVA and N.Yu. ODINTSOVA \\ Ural State Technical University, 19 Mira, Ekaterinburg 620002, Russia
}

(Received 28 January 2002; In final form 12 February 2002)

\begin{abstract}
A general scheme for the solution of problem of averaging elastic properties of textured polycrystals is suggested which is based on the algebraic methods of description of elastic properties.
\end{abstract}

Keywords: Textured polycrystals; Elastic constants; Methods of averaging

\section{INTRODUCTION}

On the assumption that grain orientations in polycrystals are equiprobable and a polycrystal as any isotropic body is characterized by two elastic constants, the problem of determination of effective elastic properties was for the first time solved by Voigt (1928) with the use of procedure of averaging the matrix of elastic modules of crystals, and then by Reuss (1929) applying the averaging of reverse matrix-matrix of the compliance coefficients. More detailed analysis performed by Hill (1952) showed that these averages agree with the assumptions on the strain uniformity in a polycrystal in the first case and the stress uniformity, in the second case, and the obtained values of the bulk module and shear module of a polycrystal represent upper and lower variational boundaries for its effective properties. The above author suggested determining of the effective elastic characteristics as a mean value obtained under the assumptions of Voigt and Reuss. For a

\footnotetext{
*Corresponding author.
} 
quasiisotropic polycrystal, these boundaries are not reached and the resulting range of their possible values can be quite wide in the case of a large anisotropy of elastic properties of a polycrystal. Further investigation consisted in searching for the effective elastic characteristics of quasiisotropic polycrystals within one or another simplified hypotheses and in trials to find a narrower range of existing values.

In 1946, Lifshits and Rosenzveig obtained the effective values in the form of the sum of modules and correlation corrections averaged by the Voigt scheme using statistic equations of the theory of polycrystalline elasticity that are solved under the assumption of low anisotropy of grains, in the problem of movements taking into account only pair correlation between random elastic modules in the neighbouring points. The method of self-consistency suggested by Hershey (1954) and Kröner (1958) represents the highest degree of obviousness. The main point of the method consists in supposition of equality of the deformation field averaged over the ensemble of anisotropic particles of random orientations embedded into a matrix with effective properties to macroscopic strain. In the framework of variational approach, Hashin and Shtrikman (1962) established a narrower range of the effective elastic properties of quasiisotropic polycrystals than that fixed by the Voigt and Reuss's boundaries. The method of renormalization of statistic equations of the elasticity theory after Fokin and Shermergor (1968) and Shermergor (1977) was applied first in the framework of singular approximation and then of generalized singular approximation to gain the effective values of elastic properties of quasiisotropic polycrystals and some composite materials, as well as narrower boundaries for their range than the Hashin-Shtrikman boundaries. Neglecting of the formal part of second derivative of the Green tensor of the equilibrium equation and preservation solely of singular part used in the method of singular approximation is equivalent to the supposition of a limiting locality of correlation functions of stochastic field of the elasticity modules of an inhomogeneous medium. The results obtained by the method of singular approximation as well as by the method of a generalized singular approximation, and Hashin-Shtrikman boundaries can be found by the method of self-consistent field as well. If the elastic characteristics of a certain reference solid is taken as the matrix elastic properties and different values are assigned to these characteristics, 
then all the solutions that meet the above methods can be obtained in analytical form. This allows to some extent ascertainment of the physical sense of the formal admissions made upon mathematical modeling.

Unexpected with respect to the common trend of the works devoted to the determination of effective properties of polycrystals was a simple method of Aleksandrov (1965) who suggested averaging of the matrix of elastic modules based on their highest invariants. From the equality of the matrix determinants for the elastic modules of a cubic crystal and isotropic body with effective properties, the value of effective shear module for quasiisotropic polycrystal was obtained. In what followed, using properties of the second rank tensor, Aleksandrov and Aisenberg (1966) revealed similarity of this way of averaging to averaging of logarithms of eigenvalues of corresponding matrix. This circumstance became then of decisive importance for the theory development. Independently of Alekseandrov, Peresada (1971) performed averaging based on the highest invariants for polycrystals of cubic and hexagonal structure symmetries.

Much more complicated than calculation of elastic properties of quasiisotropic polycrystals was the problem of their determination when there is a preferential spatial orientation of grains - texture, and on account of it, such polycrystal starts behaving as an anisotropic body. The methods of calculation of elastic characteristics of textured polycrystals developed with advancing experimental techniques of examining texture and its quantitative description.

In 1960, Wiglin suggested the method of quantitative description of textures with the use of a texture function which was then named the orientation distribution function. This function is a quantitative characteristic of distribution of random Euler angles fixing positions of individual crystallites in laboratory system of axes. The above author suggested representation of this function as a series of generalized spherical functions. Later, independently of each other, Bunge (1965) and Roe (1965) developed a method of calculating three-dimensional functions of the grain orientation distribution from the experimentally observed two-dimensional distribution functions that can formally be obtained by integrating threedimensional function. Information on even coefficients is taken quite reliably and it is these coefficients that control many physical properties 
of textured polycrystals, in particular their elastic properties. The number of significant coefficients is defined by the symmetry of grains and symmetry of the polycrystal texture.

Modern methods of quantitative texture analysis for the calculation of elastic properties of polycrystals in the approximations of Voigt, Reuss and Hill were applied by different authors (Talashkevich and Aleksandrov, 1962; Kneer, 1965; Reynolds, 1968; Bunge et al., 1969; Bunge and Roberts, 1969; Kallend and Danies, 1970; Morris, 1970). An attempt to generalize the method of calculation of the effective elastic properties after Aleksandrov-Peresada to the case of textured materials was undertaken by Morawiec (1989); Matthies and Humbert (1995). Morawiec suggested the algorithm of solution basing on the properties of the logarithmic tensor function, which was realized only in the case of a quasiisotropic material. Matthies and Humbert gave numerical realization of this algorithm for the case of some textured polycrystals that did not admit an analytical form of representation of the final solution, and the method as such was named calculation of geometric-mean values of elastic characteristics.

In the work presented an analytical generalization of the Aleksandrov-Peresada method is given for the case of textured cubic polycrystals, basing on the algebraic methods of describing their elastic properties.

\section{GENERALIZED HOOKE'S LAW AS A LINEAR APPROXIMATION}

As it was shown by Rychlewski (1984), the generalized Hooke's law can be treated as a linear self-transformation of the space of symmetrical second-rank tensors

$$
\sigma=C \varepsilon \quad \text { or } \quad \varepsilon=S \sigma .
$$

Here, $\sigma$ is the stress tensor, $\varepsilon$ is the strain tensor, $C$ is the linear elasticity operator, $S=C^{-1}$ is the reverse operator.

In the six-dimensional space of symmetrical tensors, a peculiar part is taken by the tensors that meet the equations

$$
C \omega=\lambda \omega \quad \text { or } \quad S \omega=\lambda^{-1} \omega .
$$


As in the vector spaces, in the space of symmetrical second-rank tensors there exists such an orthonormalized basis $\omega^{I}, \omega^{I I}, \ldots, \omega^{V I}$ :

$$
\omega^{K} \cdot \omega^{L}=\omega_{i j}^{K} \omega_{i j}^{L}=\delta_{K L}=\left\{\begin{array}{ll}
0 & K \neq L \\
1 & K=L
\end{array},\right.
$$

that the tensors of stresses and strains in this basis can be represented as

$$
\begin{aligned}
& \sigma=\sigma_{1} \omega^{I}+\sigma_{2} \omega^{I I}+\cdots+\sigma_{6} \omega^{V I}, \\
& \varepsilon=\varepsilon_{1} \omega^{I}+\varepsilon_{2} \omega^{I I}+\cdots+\varepsilon_{6} \omega^{V I} .
\end{aligned}
$$

The elements of tensor basis $\omega^{K}(K=1,2, \ldots, 6)$ correspond to different stress-deformed states (proper elastic states).

The fourth-rank tensor of elasticity modules $c$ matching the linear operator $C$ has the following spectral form

$$
c=\lambda_{1} \omega^{I} \otimes \omega^{I}+\lambda_{2} \omega^{I I} \otimes \omega^{I I}+\cdots+\lambda_{6} \omega^{V I} \otimes \omega^{V I},
$$

similar to the tensors of the compliance coefficients $s=c^{-1}$

$$
s=\left(\lambda_{1}\right)^{-1} \omega^{I} \otimes \omega^{I}+\left(\lambda_{2}\right)^{-1} \omega^{I I} \otimes \omega^{I I}+\cdots+\left(\lambda_{6}\right)^{-1} \omega^{V I} \otimes \omega^{V I} .
$$

Here

$$
\left(\omega^{K} \otimes \omega^{K}\right)_{i j m n}=\omega_{i j}^{K} \omega_{m n}^{K} .
$$

The parameters $\lambda_{K}(K=1,2, \ldots, 6)$ are the eigenvalues of linear operator $C$. These parameters are controlled by the elasticity modules of an anisotropic body and were called by Rychlewski true stiffness modules, and while taking into account the comments given in the work (Rychlewski, 1984) they are pertinent to call the Kelvin-Rychlewski modules. These modules are roots of the sixorder equation

$$
\operatorname{det}\left(\hat{c}_{K L}-\lambda \delta_{K L}\right)=0 \quad(K, L=1, \ldots, 6) .
$$

where

$$
\hat{c}_{K L}=\omega^{K} \cdot c \cdot \omega^{L} .
$$


The values $\hat{c}_{K L}$ (Mehrabadi and Cowin, 1990) shouldn't be confused with the matrix elements of the elasticity modules $c_{k l}$ in the Voight's designations.

Scalar multipliers $\sigma_{K}$ and $\varepsilon_{K}$ in expansion (4) with allowance for the orthogonality condition (3) for the elements of tensor's basis are determined from the expressions

$$
\begin{gathered}
\sigma_{K}=\omega^{K} \cdot \sigma \lambda(K=1,2, \ldots, 6), \\
\varepsilon_{K}=\omega^{K} \cdot \varepsilon \lambda(K=1,2, \ldots, 6) .
\end{gathered}
$$

\section{FORMAL SCHEME OF CALCULATION OF THE EFFECTIVE ELASTIC PROPERTIES OF TEXTURED POLYCRYSTALS}

In the framework of the Voight's model assuming uniformity of deformations and of the Reuss's model that suggests the stress uniformity, the effective elastic characteristics are found by averaging the tensors of elasticity modules and compliance coefficients over the set of grain orientations in a polycrystal:

$$
c^{V}=\langle c\rangle, \quad s^{R}=\langle s\rangle
$$

or with allowance for expansions (5) and (6):

$$
\begin{aligned}
c^{V}= & \lambda_{1}\langle Q\rangle *\left(\omega^{I} \otimes \omega^{I}\right)+\lambda_{2}\langle Q\rangle *\left(\omega^{I I} \otimes \omega^{I I}\right)+\cdots+\lambda_{6}\langle Q\rangle *\left(\omega^{V I} \otimes \omega^{V I}\right) \\
s^{R}= & \left(\lambda_{1}\right)^{-1}\langle Q\rangle *\left(\omega^{I} \otimes \omega^{I}\right)+\left(\lambda_{2}\right)^{-1}\langle Q\rangle *\left(\omega^{I I} \otimes \omega^{I I}\right) \\
& +\cdots+\left(\lambda_{6}\right)^{-1}\langle Q\rangle *\left(\omega^{V I} \otimes \omega^{V I}\right) .
\end{aligned}
$$

Here,

$$
\left(\langle Q\rangle *\left(\omega^{K} \otimes \omega^{K}\right)\right)_{i j m n}=\left\langle Q_{i p} Q_{j q} Q_{m r} Q_{n s}\right\rangle \omega_{p q}^{K} \omega_{r s}^{K}
$$

where $Q_{i p}$ are the elements of the transition matrix upon rotation of the crystallographic system of coordinate for a randomly oriented grain till its coincidence with the axes of the laboratory coordinate system; $\langle\cdots\rangle$ is the operation of averaging over the multitude of grain orientations in a polycrystal; $\lambda_{K}$ are the Kelvin-Rychlewski grain modules. 
On the other hand, tensors $c^{V}$ and $s^{R}$ can be presented by spectral expansions over the elements of corresponding tensor basis $\tilde{\omega}^{K}$ :

$$
\begin{aligned}
& c^{V}=\lambda_{1}^{V} \tilde{\omega}^{I} \otimes \tilde{\omega}^{I}+\lambda_{2}^{V} \tilde{\omega}^{I I} \otimes \tilde{\omega}^{I I}+\cdots+\lambda_{6}^{V} \tilde{\omega}^{V I} \otimes \tilde{\omega}^{V I}, \\
& s^{R}=\left(\lambda_{1}^{R}\right)^{-1} \tilde{\omega}^{I} \otimes \tilde{\omega}^{I}+\left(\lambda_{2}^{R}\right)^{-1} \tilde{\omega}^{I I} \otimes \tilde{\omega}^{I I}+\cdots+\left(\lambda_{6}^{R}\right)^{-1} \tilde{\omega}^{V I} \otimes \tilde{\omega}^{V I} .
\end{aligned}
$$

Comparing expansions (12) and (14) and applying the orthogonality condition (5) we find the Kelvin-Rychlewski modules in the Voight's approximation:

$$
\begin{aligned}
\lambda_{K}^{V}= & \lambda_{1}\left(\tilde{\omega}^{K} \otimes \tilde{\omega}^{K}\right) *\langle Q\rangle *\left(\omega^{I} \otimes \omega^{I}\right)+\lambda_{2}\left(\tilde{\omega}^{K} \otimes \tilde{\omega}^{K}\right) *\langle Q\rangle *\left(\omega^{I I} \otimes \omega^{I I}\right) \\
& +\cdots+\lambda_{6}\left(\tilde{\omega}^{K} \otimes \tilde{\omega}^{K}\right) *\langle Q\rangle *\left(\omega^{V I} \otimes \omega^{V I}\right) .
\end{aligned}
$$

Similarly, in the Reuss's approximation:

$$
\begin{aligned}
\left(\lambda_{K}^{R}\right)^{-1}= & \left(\lambda_{1}\right)^{-1}\left(\tilde{\omega}^{K} \otimes \tilde{\omega}^{K}\right) *\langle Q\rangle *\left(\omega^{I} \otimes \omega^{I}\right) \\
& +\left(\lambda_{2}\right)^{-1}\left(\tilde{\omega}^{K} \otimes \tilde{\omega}^{K}\right) *\langle Q\rangle *\left(\omega^{I I} \otimes \omega^{I I}\right) \\
& +\cdots+\left(\lambda_{6}\right)^{-1}\left(\tilde{\omega}^{K} \otimes \tilde{\omega}^{K}\right) *\langle Q\rangle *\left(\omega^{V I} \otimes \omega^{V I}\right) .
\end{aligned}
$$

or

$$
\begin{aligned}
\lambda_{K}^{V} & =p_{K 1} \lambda_{1}+p_{K 2} \lambda_{2}+\cdots+p_{K 6} \lambda_{6}, \\
\left(\lambda_{K}^{R}\right)^{-1} & =p_{K 1}\left(\lambda_{1}\right)^{-1}+p_{K 2}\left(\lambda_{2}\right)^{-1}+\cdots+p_{K 6}\left(\lambda_{6}\right)^{-1},
\end{aligned}
$$

where

$$
p_{K L}=\left(\tilde{\omega}^{K} \otimes \tilde{\omega}^{K}\right) *\langle Q\rangle *\left(\omega^{L} \otimes \omega^{L}\right),
$$

and, at the same time, satisfies to

$$
p_{K 1}+p_{K 2}+\cdots+p_{K 6}=1 \text {. }
$$

Thus the Kelvin-Rychlewski modules in these approximations are found as weighted average power values of corresponding modules of crystallites

$$
\lambda_{K}^{(\alpha)}=\left(p_{K 1} \lambda_{1}^{\alpha}+p_{K 2} \lambda_{2}^{\alpha}+\cdots+p_{K 6} \lambda_{6}^{\alpha}\right)^{1 / \alpha} .
$$


At $\alpha=1$, we have averaged values calculated using the Voight scheme, and at $\alpha=-1$, using the Reuss scheme. At $\alpha \rightarrow 0$, the average power value tends to the geometric mean

$$
\lambda_{K}^{(0)}=\lambda_{1}^{p_{K 1}} \lambda_{2}^{p_{K 2}} \lambda_{3}^{p_{K 3}} \lambda_{4}^{p_{K 4}} \lambda_{5}^{p_{K 5}} \lambda_{6}^{p_{K 6}},
$$

which is a generalization of the Aleksandrov-Peresada method to the textured materials.

From the formal point of view, Eq. (18) unambiguously gives the solution for the problem of averaging elastic characteristics of textured materials. Transition to the tensor designations is performed using formula (5)

$$
c^{(\alpha)}=\lambda_{1}^{(\alpha)} \tilde{\omega}^{I} \otimes \tilde{\omega}^{I}+\lambda_{2}^{(\alpha)} \tilde{\omega}^{I I} \otimes \tilde{\omega}^{I I}+\cdots+\lambda_{6}^{(\alpha)} \tilde{\omega}^{V I} \otimes \tilde{\omega}^{V I} .
$$

\section{ELASTICITY MODULES OF THE TEXTURED POLYCRYSTALS OF CUBIC SYMMETRY}

Let us consider a textured polycrystal with the cubic grain symmetry and rhombohedral texture symmetry. For an isotropic body and an anisotropic solid of cubic symmetry the elements of the tensor basis $\omega^{K}(K=1,2, \ldots, 6)$ are given in the form

$$
\begin{aligned}
\omega^{I} & =\frac{1}{3^{1 / 2}}\left(\begin{array}{ccc}
1 & 0 & 0 \\
0 & 1 & 0 \\
0 & 0 & 1
\end{array}\right), \quad \omega^{I I}=\frac{1}{6^{1 / 2}}\left(\begin{array}{ccc}
1 & 0 & 0 \\
0 & 1 & 0 \\
0 & 0 & -2
\end{array}\right), \\
\omega^{I I I} & =\frac{1}{2^{1 / 2}}\left(\begin{array}{ccc}
1 & 0 & 0 \\
0 & -1 & 0 \\
0 & 0 & 0
\end{array}\right), \quad \omega^{I V}=\frac{1}{2^{1 / 2}}\left(\begin{array}{lll}
0 & 0 & 0 \\
0 & 0 & 1 \\
0 & 1 & 0
\end{array}\right), \\
\omega^{V} & =\frac{1}{2^{1 / 2}}\left(\begin{array}{lll}
0 & 0 & 1 \\
0 & 0 & 0 \\
1 & 0 & 0
\end{array}\right), \quad \omega^{V I}=\frac{1}{2^{1 / 2}}\left(\begin{array}{lll}
0 & 1 & 0 \\
1 & 0 & 0 \\
0 & 0 & 0
\end{array}\right) .
\end{aligned}
$$

Direct checking with the use of Eq. (22) easily shows that the above elements form an ortho-normalized basis in the space of symmetrical second-rank tensors. The elements of this basis correspond to one 
stressed state of uniform compression (tension) and five stressed states of pure shears. That means that any stressed-deformed state of an anisotropic body of cubic symmetry and an isotropic body can be obtained as cumulative effect of uniform compression or tension and five shears (proper elastic states).

For the elastic characteristics $c^{V}$ and $s^{R}$ of a solid of rhombohedral symmetry, in the case when one of proper states correspond to the stressed state of uniform compression, the tensor basis elements $\tilde{\omega}^{K}(K=1, \ldots, 6)$ have the form

$$
\begin{aligned}
\tilde{\omega}^{I} & =\frac{1}{3^{1 / 2}}\left(\begin{array}{ccc}
1 & 0 & 0 \\
0 & 1 & 0 \\
0 & 0 & 1
\end{array}\right), \\
\tilde{\omega}^{I I, I I I} & =\frac{1}{\left(2+2 p_{2,3}+2 p_{2,3}^{2}\right)^{1 / 2}}\left(\begin{array}{ccc}
1 & 0 & 0 \\
0 & p_{2,3} & 0 \\
0 & 0 & -1-p_{2,3}
\end{array}\right), \\
\tilde{\omega}^{I V} & =\frac{1}{2^{1 / 2}}\left(\begin{array}{lll}
0 & 0 & 0 \\
0 & 0 & 1 \\
0 & 1 & 0
\end{array}\right), \quad \tilde{\omega}^{V}=\frac{1}{2^{1 / 2}}\left(\begin{array}{ccc}
0 & 0 & 1 \\
0 & 0 & 0 \\
1 & 0 & 0
\end{array}\right), \\
\tilde{\omega}^{V I} & =\frac{1}{2^{1 / 2}}\left(\begin{array}{lll}
0 & 1 & 0 \\
1 & 0 & 0 \\
0 & 0 & 0
\end{array}\right) .
\end{aligned}
$$

where

$$
\begin{gathered}
p_{2,3}=k \pm\left(k^{2}+k+1\right)^{1 / 2} \\
k=\left(c_{13}^{V}-c_{23}^{V}\right) /\left(c_{12}^{V}-c_{13}^{V}\right)=\left(s_{13}^{R}-s_{23}^{R}\right) /\left(s_{12}^{R}-s_{13}^{R}\right)=\left(\Delta_{1}-\Delta_{2}\right) /\left(\Delta_{2}-\Delta_{3}\right) .
\end{gathered}
$$

The parameter $k$ is the stiffness distributor which does not depend on the way of averaging and is controlled solely by the parameters of the polycrystal texture (Mityushova et al., 1985, Mityushov et al., 1992):

$$
\Delta_{i}=\left\langle Q_{i 1}^{2} Q_{i 2}^{2}+Q_{i 2}^{2} Q_{i 3}^{2}+Q_{i 3}^{2} Q_{i 1}^{2}\right\rangle(i=1,2,3) .
$$


For a nontextured material

$$
\Delta_{1}=\Delta_{2}=\Delta_{3}=1 / 5 .
$$

For a material with an axial texture

$$
\Delta_{1}=\Delta_{2}=\left(1+3 \Delta_{3}\right) / 8 .
$$

With allowance for an explicit form of tensors $\omega^{K}$ and $\tilde{\omega}^{K}$, we find from formulas (18) and (20)

$$
\begin{aligned}
\lambda_{1}^{(\alpha)}= & \lambda_{1}, \\
\lambda_{2,3}^{(\alpha)}= & \left(\lambda_{2}^{\alpha}\left(1-3 \Delta_{1}+\Delta_{2}-\Delta_{3}+2 p_{2,3}\left(\Delta_{2}-\Delta_{3}\right)\right)\right. \\
& \left.+\lambda_{4}^{\alpha}\left(3 \Delta_{1}-\Delta_{2}+\Delta_{3}-2 p_{2,3}\left(\Delta_{2}-\Delta_{3}\right)\right)\right)^{1 / \alpha}, \\
\lambda_{4}^{(\alpha)}= & \left(\lambda_{2}^{\alpha}\left(2 \Delta_{2}+2 \Delta_{3}-2 \Delta_{1}\right)+\lambda_{4}^{\alpha}\left(1-\left(2 \Delta_{2}+2 \Delta_{3}-2 \Delta_{1}\right)\right)\right)^{1 / \alpha}, \\
\lambda_{5}^{(\alpha)}= & \left(\lambda_{2}^{\alpha}\left(2 \Delta_{3}+2 \Delta_{1}-2 \Delta_{2}\right)+\lambda_{4}^{\alpha}\left(1-\left(2 \Delta_{3}+2 \Delta_{1}-2 \Delta_{2}\right)\right)\right)^{1 / \alpha}, \\
\lambda_{6}^{(\alpha)}= & \left(\lambda_{2}^{\alpha}\left(2 \Delta_{1}+2 \Delta_{2}-2 \Delta_{3}\right)+\lambda_{4}^{\alpha}\left(1-\left(2 \Delta_{1}+2 \Delta_{2}-2 \Delta_{3}\right)\right)\right)^{1 / \alpha} .
\end{aligned}
$$

The Kelvin-Rychlewski modules of a cubic crystal are expressed through the elasticity modules in the Voight designations by equalities

$$
\lambda_{1}=c_{11}+2 c_{12}=3 K, \quad \lambda_{2}=\lambda_{3}=c_{11}-c_{12}, \quad \lambda_{4}=\lambda_{5}=\lambda_{6}=2 c_{44}
$$

where $K$ is the bulk elasticity module.

The tensor of the polycrystal elasticity modules $c^{(\alpha)}$ is determined basing on expression (22).

Hence, at $\alpha=1$ and $\alpha=-1$ we find the elastic module values that were obtained in the work (Mityushov et al., 1992) by averaging over the Voight's and Reuss's schemes

$$
\begin{aligned}
c^{(1)}=c^{V}, \\
c_{11}^{V}=c_{11}-2 b \Delta_{1}, \quad c_{22}^{V}=c_{11}-2 b \Delta_{2}, \quad c_{33}^{V}=c_{11}-2 b \Delta_{3}, \\
c_{23}^{V}=c_{12}+b\left(\Delta_{2}+\Delta_{3}-\Delta_{1}\right), \quad c_{44}^{V}=c_{44}+b\left(\Delta_{2}+\Delta_{3}-\Delta_{1}\right), \\
c_{31}^{V}=c_{12}+b\left(\Delta_{3}+\Delta_{1}-\Delta_{2}\right), \quad c_{55}^{V}=c_{44}+b\left(\Delta_{3}+\Delta_{1}-\Delta_{2}\right), \\
c_{12}^{V}=c_{12}+b\left(\Delta_{1}+\Delta_{2}-\Delta_{3}\right), \quad c_{66}^{V}=c_{44}+b\left(\Delta_{1}+\Delta_{2}-\Delta_{3}\right), \\
b=c_{11}-c_{12}-2 c_{44},
\end{aligned}
$$




$$
c^{(-1)}=s^{R},
$$

$$
\begin{aligned}
& s_{11}^{R}=s_{11}-2 d \Delta_{1}, \quad s_{22}^{R}=s_{11}-2 d \Delta_{2}, \quad s_{33}^{R}=s_{11}-2 d \Delta_{3}, \\
& s_{23}^{R}=s_{12}+d\left(\Delta_{2}+\Delta_{3}-\Delta_{1}\right), \quad s_{44}^{R}=s_{44}+4 d\left(\Delta_{2}+\Delta_{3}-\Delta_{1}\right), \\
& s_{31}^{R}=s_{12}+d\left(\Delta_{3}+\Delta_{1}-\Delta_{2}\right), \quad s_{55}^{R}=s_{44}+4 d\left(\Delta_{3}+\Delta_{1}-\Delta_{2}\right), \\
& s_{12}^{R}=s_{12}+d\left(\Delta_{1}+\Delta_{2}-\Delta_{3}\right), \quad s_{66}^{R}=s_{44}+4 d\left(\Delta_{1}+\Delta_{2}-\Delta_{3}\right), \\
& d=s_{11}-s_{12}-s_{44} / 2 \text {. }
\end{aligned}
$$

At $\alpha \rightarrow 0$ geometric-mean values of the elasticity modules are found from Eq. (22)

$$
\begin{aligned}
& c_{11}^{(0)}=\frac{1}{3} \lambda_{1}^{(0)}+\frac{1}{2+2 p_{2}+2 p_{2}^{2}} \lambda_{2}^{(0)}+\frac{1}{2+2 p_{3}+2 p_{3}^{2}} \lambda_{3}^{(0)}, \\
& c_{22}^{(0)}=\frac{1}{3} \lambda_{1}^{(0)}+\frac{p_{2}}{2+2 p_{2}+2 p_{2}^{2}} \lambda_{2}^{(0)}+\frac{p_{3}}{2+2 p_{3}+2 p_{3}^{2}} \lambda_{3}^{(0)}, \\
& c_{33}^{(0)}=\frac{1}{3} \lambda_{1}^{(0)}+\frac{\left(1+p_{2}\right)^{2}}{2+2 p_{2}+2 p_{2}^{2}} \lambda_{2}^{(0)}+\frac{\left(1+p_{3}\right)^{2}}{2+2 p_{3}+2 p_{3}^{2}} \lambda_{3}^{(0)}, \\
& c_{23}^{(0)}=\frac{1}{3} \lambda_{1}^{(0)}-\frac{p_{2}\left(1+p_{2}\right)}{2+2 p_{2}+2 p_{2}^{2}} \lambda_{2}^{(0)}-\frac{p_{3}\left(1+p_{3}\right)}{2+2 p_{3}+2 p_{3}^{2}} \lambda_{3}^{(0)}, \\
& c_{31}^{(0)}=\frac{1}{3} \lambda_{1}^{(0)}+\frac{1+p_{2}}{2+2 p_{2}+2 p_{2}^{2}} \lambda_{2}^{(0)}+\frac{1+p_{3}}{2+2 p_{3}+2 p_{3}^{2}} \lambda_{3}^{(0)}, \\
& c_{12}^{(0)}=\frac{1}{3} \lambda_{1}^{(0)}+\frac{p_{2}}{2+2 p_{2}+2 p_{2}^{2}} \lambda_{2}^{(0)}+\frac{p_{3}}{2+2 p_{3}+2 p_{3}^{2}} \lambda_{3}^{(0)}, \\
& c_{44}^{(0)}=\frac{1}{2} \lambda_{4}^{(0)}, \quad c_{55}^{(0)}=\frac{1}{2} \lambda_{5}^{(0)}, \quad c_{66}^{(0)}=\frac{1}{2} \lambda_{6}^{(0)},
\end{aligned}
$$

where

$$
\begin{aligned}
& \lambda_{1}^{(0)}=\lambda_{1}, \\
& \lambda_{2,3}^{(0)}=\lambda_{2}^{\left(1-3 \Delta_{1}+\Delta_{2}-\Delta_{3}+2 p_{2,3}\left(\Delta_{2}-\Delta_{3}\right)\right)} \lambda_{4}{ }^{\left(3 \Delta_{1}-\Delta_{2}+\Delta_{3}-2 p_{2,3}\left(\Delta_{2}-\Delta_{3}\right)\right)}, \\
& \lambda_{4}^{(0)}=\lambda_{2}^{\left(2 \Delta_{2}+2 \Delta_{3}-2 \Delta_{1}\right)} \lambda_{4}{ }^{\left(1-\left(2 \Delta_{2}+2 \Delta_{3}-2 \Delta_{1}\right)\right)}, \\
& \lambda_{5}^{(0)}=\lambda_{2}^{\left(2 \Delta_{3}+2 \Delta_{1}-2 \Delta_{2}\right)} \lambda_{4}{ }^{\left(1-\left(2 \Delta_{3}+2 \Delta_{1}-2 \Delta_{2}\right)\right)}, \\
& \lambda_{6}^{(0)}=\lambda_{2}^{\left(2 \Delta_{1}+2 \Delta_{2}-2 \Delta_{3}\right)} \lambda_{4}{ }^{\left(1-\left(2 \Delta_{1}+2 \Delta_{2}-2 \Delta_{3}\right)\right)} .
\end{aligned}
$$


Some particular results of these relationships were obtained earlier by other methods. Thus, the Aleksandrov's solution (1965) is resulted at $\Delta_{1}=\Delta_{2}=\Delta_{3}=1 / 5$. For shear modules, the generalization of this solution was given in the work (Mityushova, 1983). In the case of axial textures, the solution was presented in the work (Mityushov and Berestova, 1996); and for a polycrystal with a two-component texture $(001)[100]+(001)[110]$ when $\Delta_{1}=\Delta_{2}=1 / 4, \quad \Delta_{3}=0$, this solution is precise (Berestova and Mityushov, 1999).

\section{References}

Aleksandrov, K.S. (1965). Means of tensor values. Dokl. A. N. SSSR, 164, 800-803.

Aleksandrov, K.S. and Aizenberg, L.A. (1966). Method for determining physical constants of polycrystal materials. Dokl. A. N. SSSR, 167, 1028-1031.

Berestova, S.A. and Mityushov, E.A. (1999). An exact determination of the effective moduli of elasticity of micro-ingomogeneous media. J. Appl. Maths. Mechs., 63, 524-527.

Bunge, H. J. (1965). Zur darstellung allgemeiner texturen. J. Metallk., 56, 872-874.

Bunge, H.J., Ebert, R. and Günther, F. (1969a). Of the angular variation and texture dependence of Young's modulus in cold-rolled copped sheet. Phys. Stat. Sol., 31, 565 .

Bunge, H.J. and Roberts, W.T. (1969b). Orientation distribution elastic and plastic anisotropie in stabilized steel. J. Appl. Cryst., 2, 116.

Fokin, A.G. and Shermergor, T.D. (1968). Boundaries for effective modules of ingomogeneous solids. Zh. Prikl. Mekh. Tech. Fis., 4, 39.

Hashin Z. and Shtrikman S. (1962). On gomt variational principles in anisotropic and nonhomogeneous elasticity. J. Mech. and Plysh. Solids, 10, 343.

Hershey, A.V. (1954).The elasticity of an isotropic aggregate anisotropic cubic crystals. J. Appl.Mech., 21, 236-242.

Hill, R. (1952). The elastic behaviour of a crystalline aggregate. Proc. Phys. Soc., (A) 65, 349-354.

Kallend, J.S. and Davies, G.J. (1970). The prediction of plastic anisotropy in annealed sheets of copper and $\alpha$-brass. J. Inst. Met., 98, 242-244.

Kneer, G. (1965). Uber Berechnung der elastizitatsmodulu vielkristalliner aggregate mit texture. Phys. Status Solidi, 9, K825-838.

Krőner, E. (1958). Berechnung der elastischen konstanten vielkristalls aus der konstanten des einkristalls. Z. Phys., 151, 504-518.

Lifshits, I.M. and Rosenzveig, L.N. (1946). The theory of the elastic properties of polycrystals. Zh. Eksp. Teor. Fis., 16, 967-980.

Matthies, S. and Humbert, M. (1995). On the principle of geometric mean of even-rank symmetric tensors for textured polycrystals. J. Appl. Cryst., 28, 254-266.

Mehrabadi, M.M. and Cowin, S.C. (1990). Eigentensors of linear anisotropic elastic materials. Mech. Appl. Math., 43, 15-41.

Mityushov, E.A. and Berestova, S.A., (1996). The geometric mean of elastic constants for textured polycrystals. Proc. ICOTOM-11, pp. 830-835.

Mityushov, E.A., Gel'd, P.V. and Adamesku, R.A. (1992). Generalized Conductivity and Elasticity of Macrouniform Heterogeneous Materials. Metallurgy, Moscow. 
Mityushova, L.L., Mityushov, E.A., Adamesku, R.A. and Yushkov V.I. (1985). Orientation factors of anysotropy of elastic properties for cubic metals. Fiz. Met. Metalloved, 60, 993-995.

Mityushova, L.L. (1983). Elastic and plastic anisotropy of textured polycrystals of cubic syngony. Thesis for candidate degree of physics and mathematics. Ural Polytechnical Institute, Sverdlovsk.

Morawiec, A. (1989). Calculation of polycrystal elastic constants. Phys. Stat. Sol., (b) 154, 535-541.

Morris, P.R. (1970). Elastic constants of polycrystals. Int. J. Eng. Sci., 8, 49-55.

Peresada, G.I. (1971). On the calculation of elastic moduli of polycrystalline systems from single crystal data. Phys. Stat. Sol., (a) 4, K23-K27.

Reuss, A. (1929). Berechnund der fliebgrenze von misch-kristallen fut grund der plastizitatsbedingung fur einkristalle. Z. Angew. Math. Mech., 9, 49-54.

Reynolds, W.N. (1968). Uniaxial textures in cubic materials. Philos. Mag., 18, $1155-1159$.

Roe, R.J. (1965). Description of crystallite orientation in polycrystalline materials. III. General solution to pole figure inversion. J. Appl. Phys., 36, 2024-2031.

Rychlewski, J. (1984). On Hooke's law. J. Appl . Maths. Mechs., 48, 420-435.

Shermergor, T.D. (1977). The Theory of Microinhomogeneous Media, Moscow, Nauka.

Talashkevich, I.P. and Aleksandrov, K.S. (1962). Influence of texture on elastic properties of polycrystals. Fiz. Met. Metalloved, 14, 801-805.

Voigt, W. (1928). Lehrbuch der Kristallphysik, Teubner Verlaggeselschaft, Stutgart.

Wiglin, A.S. (1960). Quantitative description of textures for polycrystal material. Physics of solids, 2, 2463-2476. 